Vietnam Journal of Mechanics, VAST, Vol.38, No. 3 (2016), pp. 181 - 192

DOI:10.15625/0866-7136/38/3/6055

\title{
IMPROVED ESTIMATES FOR THE EFFECTIVE ELASTIC BULK MODULUS OF RANDOM TETRAGONAL CRYSTAL AGGREGATES
}

\author{
Vuong Thi My Hanh, Pham Duc Chinh, Vu Lam Dong* \\ Institute of Mechanics, Vietnam Academy of Science and Technology, Hanoi, Vietnam \\ *E-mail: vldong@imech.ac.vn \\ Received April 10, 2015
}

\begin{abstract}
Particular expressions of upper and lower estimates for the macroscopic elastic bulk modulus of random cell tetragonal polycrystalline materials are derived and computed for a number of practical crystals. The cell-shape-unspecified bounds, based on minimum energy principles and generalized polarization trial fields, appear close to the simple bounds for specific spherical cell polycrystals.

Keywords: Variational bounds, effective elastic bulk modulus, random cell polycrystal, tetragonal crystal.
\end{abstract}

\section{INTRODUCTION}

Macroscopic (effective) elastic moduli of polycrystalline materials depend on the elastic constants of the base crystal and aggregates' microstructure, which is often of random and irregular nature. The first simple estimations for the effective moduli are Voigt arithmetic average and Reuss harmonic average. Using minimum energy and complimentary energy principles and constant strain and stress trial fields, respectively, Hill [1] established that Voigt and Reuss averages are upper and lower bounds on the possible values of the effective elastic moduli of orientation-unpreferable polycrystalline aggregates. Assuming that the shape and crystalline orientations of the grains within a random polycrystalline aggregates are uncorrelated and using their own variational principles, Hashin and Shtrikman [2] derived the respective second order bounds for the moduli that are significantly tighter than the first order Voigt-Reuss-Hill bounds. Using HashinShtrikman-type polarization trial fields, but comming directly from classical minimum energy principles, Pham [3-6] succeeded in constructing partly third order bounds on elastic moduli of random cell polycrystals, which fall strictly inside the second order Hashin-Shtrikman bounds. More general polarization trial fields have been used to derive even tighter bounds (still being partly third order ones, though) in [7], which are

(C) 2016 Vietnam Academy of Science and Technology 
specified to the cubic crystals' case. In this work we resume the approaches and derive the explicit expressions of the estimates for the effective bulk modulus of tetragonal polycrystals, with particular numerical results for a number of materials.

\section{MINIMUM ENERGY PRINCIPLES AND THE BOUNDS}

Consider a representative volume element $V$ of a polycrystalline aggregate that consists of $N$ components occupying regions $V_{\alpha} \subset V$ of equal volumes $v_{\alpha}=v_{0}(\alpha=$ $1, \ldots, N$ ) - each component is composed of grains of the same crystalline orientation with respective crystal elastic stiffness tensor $\mathbf{C}(\mathbf{x})=\mathbf{C}^{\alpha}$. A random cell polycrystal (see Fig. 1) is supposed to be represented by such $N$-component configuration when $N \rightarrow$ $\infty, v_{\alpha}=v_{0}=\frac{1}{N} \rightarrow 0$ with the crystalline orientations being distributed uniformly in all directions in the space. The effective elastic tensor $\mathbf{C}^{e f f}=\mathbf{T}\left(K^{e f f}, \mu^{e f f}\right)$ of the polycrystal is defined via the minimum energy expression $[3,4]$

$$
\mathcal{\varepsilon}^{0}: \mathbf{C}^{e f f}: \varepsilon^{0}=\inf _{\langle\boldsymbol{\varepsilon}\rangle=\mathcal{\varepsilon}^{0}} \int_{V} \varepsilon: \mathbf{C}: \varepsilon d \mathbf{x},
$$

or the minimum complementary energy expression

$$
\sigma^{0}:\left(\mathbf{C}^{e f f}\right)^{-1}: \sigma^{0}=\inf _{\langle\boldsymbol{\sigma}\rangle=\sigma^{0}} \int_{V} \sigma: \mathbf{C}^{-1}: \sigma d \mathbf{x},
$$

where the admissible (compatible) strain field $\varepsilon(\mathbf{x})$ in (1) is expressed through a displacement field $\mathbf{u}(\mathbf{x})$ in $V$

$$
\varepsilon=\frac{1}{2}\left[\nabla \mathbf{u}+(\nabla \mathbf{u})^{T}\right]
$$

while the stress field $\sigma(\mathbf{x})$ in (2) is equilibrated in $V$

$$
\nabla \cdot \sigma=\mathbf{0}
$$

$\langle\cdot\rangle$ means the volume average on $V ; \varepsilon^{0}, \sigma^{0}$ are constant strain and stress fields; $\mathbf{T}(K, \mu)$ are isotropic fourth rank tensor function

$$
T_{i j k l}(K, \mu)=K \delta_{i j} \delta_{k l}+\mu\left(\delta_{i k} \delta_{j l}+\delta_{i l} \delta_{j k}-\frac{2}{3} \delta_{i j} \delta_{k l}\right) \text {. }
$$

The strain and stress fields are related via the Hook law $\sigma(\mathbf{x})=\mathbf{C}(\mathbf{x}): \varepsilon(\mathbf{x})$.

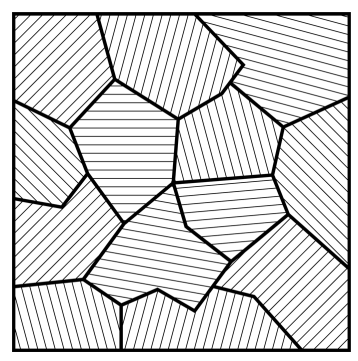

Fig. 1. A random cell polycrystal 
In [7], the admissible multi-parameter kinematic and static polarization trial fields more general than those in [3-6], which have contain only two free parameters, have been chosen

$$
\varepsilon_{i j}(\mathbf{x})=\varepsilon^{0} \delta_{i j}+\sum_{\alpha=1}^{N}\left[a_{i k}^{\alpha} \varphi_{, k j}^{\alpha}+a_{j k}^{\alpha} \varphi_{, k i}^{\alpha}+b a_{k l}^{\alpha} \psi_{, i j k l}^{\alpha}\right]
$$

for (1), and

$$
\sigma_{i j}(\mathbf{x})=\sigma^{0} \delta_{i j}+\sum_{\alpha=1}^{N}\left[a_{i k}^{\alpha} \varphi_{, k j}^{\alpha}+a_{j k}^{\alpha} \varphi_{, k i}^{\alpha}-(b+1) \delta_{i j} a_{k l}^{\alpha} \varphi_{, k l}^{\alpha}-a_{i j}^{\alpha} I^{\alpha}+b a_{k l}^{\alpha} \psi_{, i j k l}^{\alpha}\right],
$$

for (2), where $\mathcal{I}^{\alpha}(\mathbf{x})$ equals to 1 if $\mathbf{x} \in V_{\alpha}$, and to 0 if $\mathbf{x} \notin V_{\alpha}$; and we have introduced the harmonic and biharmonic potentials

$$
\begin{gathered}
\varphi^{\alpha}(\mathbf{x})=-\int_{V_{\alpha}} \frac{1}{4 \pi}|\mathbf{x}-\mathbf{y}|^{-1} d \mathbf{y}, \quad \psi^{\alpha}(\mathbf{x})=-\int_{V_{\alpha}} \frac{1}{8 \pi}|\mathbf{x}-\mathbf{y}| d \mathbf{y}, \\
\left(\nabla^{2} \varphi^{\alpha}(\mathbf{x})=\nabla^{4} \psi^{\alpha}(\mathbf{x})=\delta_{\alpha \beta}, \quad \mathbf{x} \in V_{\beta}\right) .
\end{gathered}
$$

The free parameters $a_{i k}^{\alpha}$ in Eqs. (6) and (7) are subjected to restrictions (for $\left\langle\varepsilon_{i j}\right\rangle=$ $\left.\varepsilon^{0} \delta_{i j},\left\langle\sigma_{i j}\right\rangle=\sigma^{0} \delta_{i j}\right)$

$$
\sum_{\alpha=1}^{N} v_{\alpha} a_{i k}^{\alpha}=0, \quad i, k=1,2,3 .
$$

Substituting the trial fields (6) and (7) into the energy functionals of (1) and (2) , and optimizing the respective energy functions over free parameters $a_{i k}^{\alpha}$ restricted by Eq. (9), with the help of Lagrange multipliers, and then over $b$, one obtains the formal bounds on $K^{e f f}[7]$

$$
K^{U} \geq K^{e f f} \geq K^{L}
$$

where

$$
\begin{aligned}
& K^{U}(\mathbf{C})=\max _{f_{1}, g_{1} \in(12)} \min _{b} K^{U f g}\left(\mathbf{C}, f_{1}, g_{1}, b\right), \\
& K^{U f g}=k_{V}+\left\langle\mathbf{C}_{K \alpha}: \mathcal{A}_{\alpha}^{-1}\right\rangle_{\alpha}:\left\langle\mathcal{A}_{\alpha}^{-1}\right\rangle_{\alpha}^{-1}:\left\langle\mathcal{A}_{\alpha}^{-1}: \mathbf{C}_{K \alpha}\right\rangle_{\alpha}-\left\langle\mathbf{C}_{K \alpha}: \mathcal{A}_{\alpha}^{-1}: \mathbf{C}_{K \alpha}\right\rangle_{\alpha}, \\
& K^{L}(\mathbf{C})=\min _{f_{1}, g_{1} \in(12)} \max _{b} K^{L f g}\left(\mathbf{C}, f_{1}, g_{1}, b\right), \\
& K^{L f g}=\left(k_{R}^{-1}+\left\langle\overline{\mathbf{C}}_{K \alpha}: \overline{\mathcal{A}}_{K \alpha}^{-1}\right\rangle_{\alpha}:\left\langle\overline{\mathcal{A}}_{\alpha}^{-1}\right\rangle_{\alpha}^{-1}:\left\langle\overline{\mathcal{A}}_{\alpha}^{-1}: \overline{\mathbf{C}}_{\alpha}\right\rangle_{\alpha}-\left\langle\overline{\mathbf{C}}_{K \alpha}: \overline{\mathcal{A}}_{\alpha}^{-1}: \overline{\mathbf{C}}_{K \alpha}\right\rangle_{\alpha}\right)^{-1,},
\end{aligned}
$$

the cell (grain) shape parameters $f_{1}, g_{1}$ are restricted in the ranges

$$
\frac{2}{3} \geq f_{1} \geq 0, \quad \frac{6}{7} f_{1}+\frac{8}{35} \geq g_{1} \geq \frac{6}{7} f_{1}, \quad\left(f_{3}=\frac{2}{3}-f_{1}, g_{3}=\frac{4}{5}-g_{1}\right),
$$

$K_{V}$ and $K_{R}$ are Voigt and Reuss averages

$$
K_{V}=\frac{1}{9} C_{i i j j}, \quad K_{R}=\left[\left(\mathbf{C}^{-1}\right)_{i i j j}\right]^{-1},
$$

$\langle\cdot\rangle_{\alpha}$ designates an average over all crystalline orientations $\alpha$; the expressions of $\mathcal{A}_{\alpha}, \overline{\mathcal{A}}_{\alpha}$, $\mathbf{C}_{K \alpha}, \overline{\mathbf{C}}_{K \alpha}$, are given in Appendix A. The shape parameters $f_{1}, g_{1}$ defined in [4] contain 
three-point correlation information about the symmetric cell geometry of the random polycrystal.

In the procedure briefly presented above, substitution of the trial field (6) [or (7)] into the energy expressions (1) [or (2)] is rather involved, which required statistical symmetry and isotropy hypotheses for random cell polycrystals and much algebraic manipulation $[3,4,7]$. Still, as one can see it directly, the resulted energy expression should be a quadratic form for the free parameters $a_{i k}^{\alpha}$, which are restricted by (9). Hence, the substituted energy expression can be optimized over the free parameters analytically by the Lagrange multiplier method. The energy expression obtained still contain 3 parameters, including the free one $b$ and 2 geometric parameters $f_{1}, g_{1}$ characterizing polycrystal microstructure and restricted by (12). The last optimization operation over these 3 parameters in (11) have to be made numerically for particular cases, as shall be done subsequently. The bounds will be specified for the random aggregate of tetragonal crystals in the next section.

\section{BOUNDS FOR TETRAGONAL CRYSTAL AGGREGATES}

In the following, $C_{i j k l}(i, j, k, l=1,2,3)$ are designated as the components of the fourth-rank elastic stiffness tensor $\mathbf{C}$ in the base-crystal coordinates, and $C_{p q}(p, q=$ $1, \ldots, 6)$ are the respective convenient Voigt's two-index notations for the elastic constants. In the case of tetragonal crystals (classes $4, \overline{4}, 4 / \mathrm{m}$ ), there are 7 independent elastic constants $C_{11}, C_{12}, C_{13}, C_{33}, C_{44}, C_{16}, C_{66}$. The correspondence between the fourth-rank elasticity tensor components in the base crystal reference $C_{i j k l}$ and those in the two-index notation is

$$
\begin{aligned}
& C_{11}=C_{1111}=C_{2222}, C_{33}=C_{3333}, C_{12}=C_{1122}, C_{16}=C_{1112}=-C_{2212}, \\
& C_{13}=C_{1133}=C_{2233}, C_{44}=C_{1313}=C_{2323}, C_{66}=C_{1212},
\end{aligned}
$$

while the other independent components are zero. The elastic compliance tensor $\mathbf{S}=\mathbf{C}^{-1}$ is often given in the respective Voigt's two-index notation $S_{p q}(p, q=1, \ldots, 6)$ as

$$
\begin{aligned}
& S_{11}=S_{1111}, S_{33}=S_{3333}, S_{12}=S_{1122}, S_{16}=2 S_{1112}, \\
& S_{13}=S_{1133}, S_{44}=4 S_{2323}, S_{66}=4 S_{1212} .
\end{aligned}
$$

As such, the $6 \times 6$ matrix $\left\{S_{p q}\right\}$ is really the inverse to the matrix $\left\{C_{p q}\right\}$, with particular relations between their components

$$
\begin{aligned}
& \left\{S_{p q}\right\}=\left\{C_{p q}\right\}^{-1}, \\
& S_{11}=\frac{1}{2}\left[\frac{C_{33}}{C_{33}\left(C_{11}+C_{12}\right)-2 C_{13}^{2}}+\frac{C_{66}}{C_{66}\left(C_{11}-C_{12}\right)-2 C_{16}^{2}}\right], \\
& S_{12}=\frac{1}{2}\left[\frac{C_{33}}{C_{33}\left(C_{11}+C_{12}\right)-2 C_{13}^{2}}-\frac{C_{66}}{C_{66}\left(C_{11}-C_{12}\right)-2 C_{16}^{2}}\right], \\
& S_{13}=\frac{-C_{13}}{\left(C_{11}+C_{12}\right) C_{33}-2 C_{13}^{2}}, \quad S_{33}=\frac{C_{11}+C_{12}}{\left(C_{11}+C_{12}\right) C_{33}-2 C_{13}^{2}}, \\
& S_{44}=\frac{1}{C_{44}}, \quad S_{66}=\frac{C_{11}-C_{12}}{\left(C_{11}-C_{12}\right) C_{66}-2 C_{16}^{2}}, \quad S_{16}=\frac{-C_{16}}{\left(C_{11}-C_{12}\right) C_{66}-2 C_{16}^{2}} .
\end{aligned}
$$


The Voigt and Reuss averages have the particular expressions

$$
\begin{aligned}
& K_{V}=\mathcal{K}_{V}\left(\left\{C_{p q}\right\}\right)=\frac{1}{9}\left(2 C_{11}+2 C_{12}+4 C_{13}+C_{33}\right), \\
& K_{R}=\mathcal{K}_{R}\left(\left\{S_{p q}\right\}\right)=\left(2 S_{11}+2 S_{12}+4 S_{13}+S_{33}\right)^{-1} .
\end{aligned}
$$

Inverse (16) and functional (17) relations shall be used repeatedly afterwards.

Let the tensors $\mathrm{C}_{K \alpha}, \mathcal{A}_{\alpha}, \overline{\mathbf{C}}_{K \alpha}, \overline{\mathcal{A}}_{\alpha}$ be given as $\mathrm{C}_{K}, \mathcal{A}, \overline{\mathbf{C}}_{K}, \overline{\mathcal{A}}$ in the base crystal coordinates. Since the crystalline orientations $\alpha$ are distributed equally over all directions in the random polycrystalline material space, all the averages $\langle\cdot\rangle_{\alpha}$ in Eqs. (11) are tensor invariants, hence can be calculated in the base-crystal coordinates. For the upper bound $K^{U}$, we derive the following particular expressions of its constituent tensors as two-index-notation matrices

$$
\begin{aligned}
C_{K}= & \left.\left\{C_{i j}^{K}\right\}, \quad \text { (symmetric 3x3 matrix }\right), \\
C_{11}^{K}= & \left(C_{11}+C_{12}+C_{13}\right) \frac{1}{9}\left(1+\frac{2 b}{5}\right)+\frac{b K_{V}}{5}=C_{22}^{K}, \\
C_{33}^{K}= & \left(C_{33}+2 C_{13}\right) \frac{1}{9}\left(1+\frac{2 b}{5}\right)+\frac{b K_{V}}{5}, \quad C_{12}^{K}=C_{13}^{K}=C_{23}^{K}=0, \\
\mathcal{A}=\{ & \left.C_{p q}^{A}\right\}, \quad C_{p q}^{A}=C_{p q}^{\prime A}+D_{p q}, \quad(\text { symmetric } 6 \times 6 \text { matrices }), \\
C_{11}^{\prime A}= & C_{11}\left(B_{1}+B_{2}\right)+\left(C_{11}+C_{12}+C_{13}\right)\left(B_{3}+B_{6}\right) \\
& +\left(C_{11}+C_{44}+C_{66}\right)\left(B_{4}+B_{5}\right)=C_{22}^{\prime A}, \\
C_{33}^{\prime A}= & C_{33}\left(B_{1}+B_{2}\right)+\left(C_{33}+2 C_{13}\right)\left(B_{3}+B_{6}\right)+\left(C_{33}+2 C_{44}\right)\left(B_{4}+B_{5}\right), \\
C_{12}^{\prime A}= & C_{12} B_{1}+C_{66} B_{2}+\left(C_{11}+C_{12}+C_{13}\right) B_{3}+\left(C_{11}+C_{44}+C_{66}\right) B_{4}, \\
C_{13}^{\prime A}= & C_{13} B_{1}+C_{44} B_{2}+\left(C_{11}+C_{33}+C_{12}+3 C_{13}\right) \frac{1}{2} B_{3} \\
& +\left(C_{11}+C_{33}+3 C_{44}+C_{66}\right) \frac{1}{2} B_{4}=C_{23}^{\prime A}, \\
C_{44}^{\prime A}= & C_{44} B_{1}+\left(C_{13}+C_{44}\right) \frac{1}{2} B_{2}+\left(C_{11}+C_{33}+3 C_{44}+C_{66}\right) \frac{1}{4} B_{5} \\
& +\left(C_{11}+C_{12}+3 C_{13}+C_{33}\right) \frac{1}{4} B_{6}=C_{55}^{\prime A}, \\
C_{66}^{\prime A}= & C_{66} B_{1}+\left(C_{12}+C_{66}\right) \frac{1}{2} B_{2}+\left(C_{11}+C_{44}+C_{66}\right) \frac{1}{2} B_{5} \\
& +\left(C_{11}+C_{12}+C_{13}\right) \frac{1}{2} B_{6}, \\
C_{26}^{\prime A}= & -C_{16}^{\prime A}=-C_{16}\left(B_{1}+B_{2}\right), \\
D_{11}= & D_{22}=D_{33}=D_{1}+D_{25}, D_{12}=D_{13}=D_{23}=D_{1}, \\
D_{46}=\frac{1}{2} D_{2}, & D_{55},
\end{aligned}
$$


where $B_{1}, \ldots, B_{6}$ and $D_{1}, D_{2}$ are defined in Appendix A. Further, one finds the $6 \times 6$ symmetric matrix

$$
\mathcal{A}^{-1}=\left\{S_{p q}^{A}\right\}=\left\{C_{p q}^{A}\right\}^{-1} \quad \text { [according to relations (14)] }
$$

Then

$$
\begin{aligned}
& \mathcal{A}^{-1}: \mathbf{C}_{K}=\mathbf{C}_{K}: \mathcal{A}^{-1}=\left\{C_{K i j}^{A C}\right\} \quad(3 \times 3 \text { symmetric matrix }), \\
& C_{K 11}^{A C}=\left(S_{11}^{A}+S_{12}^{A}\right) C_{11}^{K}+S_{13}^{A} C_{33}^{K}=C_{K 22}^{A C}, \\
& C_{K 33}^{A C}=S_{33}^{A} C_{33}^{K}+2 S_{13}^{A} C_{11}^{K}, \quad C_{K 12}^{A C}=C_{K 13}^{A C}=C_{K 23}^{A C}=0,
\end{aligned}
$$

and

$$
\mathbf{C}_{K}: \mathcal{A}^{-1}: \mathbf{C}_{K}=C_{K}^{C A C}=2 C_{11}^{K} C_{K 11}^{A C}+C_{33}^{K} C_{K 33}^{A C} .
$$

Now one has [isotropic tensor $\mathbf{T}$ is defined in Eq. (5), functionals $\mathcal{K}_{R}$ - in Eq. (17)]

$$
\left\langle\mathcal{A}_{\alpha}^{-1}\right\rangle_{\alpha}=\mathbf{T}\left(\frac{1}{9} \mathcal{K}_{R}^{-1}\left(\left\{S_{p q}^{A}\right\}\right), \frac{1}{4} \mathcal{M}_{R}^{-1}\left(\left\{S_{p q}^{A}\right\}\right)\right) .
$$

Hence

$$
\left\langle\mathcal{A}_{\alpha}^{-1}\right\rangle_{\alpha}^{-1}=\mathbf{T}\left(\mathcal{K}_{R}\left(\left\{S_{p q}^{A}\right\}\right), \mathcal{M}_{R}\left(\left\{S_{p q}^{A}\right\}\right)\right)
$$

Also

$$
\left\langle\mathcal{A}_{\alpha}^{-1}: \mathbf{C}_{K \alpha}\right\rangle_{\alpha}=\left\langle\mathbf{C}_{K \alpha}: \mathcal{A}_{\alpha}^{-1}\right\rangle_{\alpha}=\mathbf{I} \frac{1}{3}\left(2 C_{K 11}^{A C}+C_{K 33}^{A C}\right),
$$

I is the second rank unit tensor. Finally

$$
K^{U f g}=K_{V}+\left(2 C_{K 11}^{A C}+C_{K 33}^{A C}\right)^{2} \mathcal{K}_{R}\left(\left\{S_{p q}^{A}\right\}\right)-C_{K}^{C A C} .
$$

The upper bound $K^{U}$ is found from expression (28) and respective optimizing operation in (11).

Similarly, for the lower bound $K^{L}$ in (11), we find

$$
K^{L f g}=\left[K_{R}^{-1}+\frac{1}{9}\left(\overline{2} C_{K 11}^{A C}+\bar{C}_{K 33}^{A C}\right)^{2} \mathcal{K}_{V}^{-1}\left(\left\{\bar{C}_{p q}^{A}\right\}\right)-\bar{C}_{K}^{C A C}\right]^{-1},
$$

where the expressions of $\bar{C}_{p q}^{A}, \bar{C}_{K p q}^{A C}, \bar{C}_{K}^{C A C}$ are given in Appendix $\mathrm{B}$, functional $\mathcal{K}_{V}$ is defined in Eq. (17). The lower bound $K^{L}$ is found from expression (29) and respective optimizing operation in (11).

For numerical calculations, we take the tetragonal crystals, elastic constants of which are collected in [8]. The new shape-unspecified bounds $K^{U}, K^{L}$ are compared with the old bounds $\hat{K}^{U}, \hat{K}^{L}$ of [5], and also with bounds for specific spherical cell polycrystals $K_{s}^{U}, K_{s}^{L}$ and $\mu_{s}^{U}, \mu_{s}^{L}$, where the shape parameters $f_{1}=g_{1}=0$, in Tab. 1 . In Tab. 2 the respective values of $b, f_{1}, g_{1}$, where the optimal bounds are reached, are also reported. It is interesting to observe that, in the case of spherical cell polycrystals, the new bounds coincide with those obtained from our old approach [5], which have simple expressions. The new shape-unspecified bounds are closer to the bounds for spherical cell polycrystals compared to the old ones. Unfortunately, up to present, we can not find in the literature any sufficiently-high-accurate experiments on the elastic moduli of random polycrystals, 
Table 1. The new shape-unspecified bounds $K^{L}, K^{U}$ on the effective bulk modulus of some tetragonal crystal aggregates compared to the old bounds $K_{\text {old }}^{L}, K_{\text {old }}^{U}$ and the bounds for spherical cell polycrystals $K_{s}^{L}, K_{s}^{U}$ (all in GPa)

\begin{tabular}{|c|c|c|c|c|c|c|}
\hline Crystal & $K_{\text {old }}^{L}$ & $K^{L}$ & $K_{s}^{L}$ & $K_{s}^{U}$ & $K^{U}$ & $K_{\text {old }}^{U}$ \\
\hline $\mathrm{C}\left(\mathrm{CH}_{3} \mathrm{OH}\right)_{4}$ & 15.23 & 15.26 & 15.41 & 16.53 & 16.58 & 16.60 \\
$\mathrm{AgClO}_{3}$ & 35.31 & 35.32 & 35.32 & 35.33 & 35.33 & 35.33 \\
$\mathrm{SrMoO}_{4}$ & 69.86 & 69.86 & 69.86 & 69.88 & 69.88 & 69.88 \\
$\mathrm{C}_{14} \mathrm{H}_{8} \mathrm{O}_{4}$ & 4.114 & 4.126 & 4.128 & 4.157 & 4.198 & 4.199 \\
$\mathrm{CaMoO}_{4}$ & 80.92 & 80.92 & 80.93 & 80.94 & 80.94 & 80.94 \\
\hline
\end{tabular}

Table 2. $b^{L}, f_{1}^{L}, g_{1}^{L}, b^{U}, f_{1}^{U}, g_{1}^{U}$ - the values of the free $(b)$ and shape $\left(f_{1}, g_{1}\right)$ parameters, at which the respective extrema in the bounds on the effective bulk modulus of the random polycrystals of Tab. 1 are reached

\begin{tabular}{|c|c|c|c|c|c|c|}
\hline Crystal & $b^{L}$ & $f_{1}^{L}$ & $g_{1}^{L}$ & $b^{U}$ & $f_{1}^{U}$ & $g_{1}^{U}$ \\
\hline $\mathrm{C}\left(\mathrm{CH}_{3} \mathrm{OH}\right)_{4}$ & -1.6020 & $2 / 3$ & 0.5714 & -0.7875 & 0 & 0.2286 \\
$\mathrm{AgClO}_{3}$ & -1.6070 & 0 & 0.2286 & -0.7928 & 0 & 0 \\
$\mathrm{SrMoO}_{4}$ & -1.4143 & $2 / 3$ & 0.5714 & -0.7086 & 0 & 0 \\
$\mathrm{C}_{14} \mathrm{H}_{8} \mathrm{O}_{4}$ & -0.8619 & 0 & 0.2286 & -0.5212 & $2 / 3$ & 0.5714 \\
$\mathrm{CaMoO}_{4}$ & -1.3817 & $2 / 3$ & 0.5714 & -0.7025 & 0 & 0 \\
\hline
\end{tabular}

in which all experimental data points are collected, not just the rough average one - with just 2 significant digits, for comparison with the bounds. Still, some data collected in [6] seem to support the prediction of our bounds.

\section{CONCLUSION}

In this paper we have derived explicit expressions of the general shape-unspecified upper and lower bounds on the effective elastic bulk modulus of random tetragonal crystal aggregates with numerical illustrations. The estimates are expected to predict the scatter range of the macroscopic elastic property of practical polycrystalline materials. One can see that the much more general and flexible trial fields (compared to the old ones of [5]) lead only to small improvements indicates again that we might close to the best possible estimates. High-accuracy experiments and numerical simulations on random polycrystals are expected to access the practical value of our theoretical results. Estimates for the more-complex effective shear modulus of the tetragonal crystal aggregates shall be the subject of our following study.

\section{ACKNOWLEDGEMENT}

This work is funded by Vietnam's National Foundation for Science and Technology Development, Project N. 107.02-2013.20. 


\section{REFERENCES}

[1] R. Hill. The elastic behaviour of a crystalline aggregate. Proceedings of the Physical Society, Section A, 65, (5), (1952), pp. 349-354.

[2] Z. Hashin and S. Shtrikman. A variational approach to the theory of the elastic behaviour of polycrystals. Journal of the Mechanics and Physics of Solids, 10, (4), (1962), pp. 343-352.

[3] D. C. Pham. Bounds on the effective shear modulus of multiphase materials. International Journal of Engineering Science, 31, (1), (1993), pp. 11-17.

[4] D. C. Pham. New estimates for macroscopic elastic moduli of random polycrystalline aggregates. Philosophical Magazine, 86, (2), (2006), pp. 205-226.

[5] D. C. Pham. Macroscopic uncertainty of the effective properties of random media and polycrystals. Journal of Applied Physics, 101, (2), (2007). Doi:10.1063/1.2426378.

[6] D. C. Pham. On the scatter ranges for the elastic moduli of random aggregates of general anisotropic crystals. Philosophical Magazine, 91, (4), (2011), pp. 609-627.

[7] D. C. Pham. Bounds on the elastic moduli of statistically isotropic multicomponent materials and random cell polycrystals. International Journal of Solids and Structures, 49, (18), (2012), pp. 2646-2659.

[8] H. H. Landolt and R. Börnstein. Group III:: Crystal and solid state physics, Vol. 11. SpringerVerlarg, (1979). 


\section{APPENDIX A}

General formula for the tensors $\mathcal{A}_{\alpha}, \overline{\mathcal{A}}_{\alpha}, \mathbf{C}_{K \alpha}, \overline{\mathbf{C}}_{K \alpha}$ appeared in Eqs. (11) of the bounds on the effective bulk modulus of random cell polycrystals [7]

$$
\begin{aligned}
& \mathbf{C}_{K \alpha}=\left\{C_{i j}^{K \alpha}\right\}, \quad C_{i j}^{K \alpha}=C_{i j k k}^{\alpha} \frac{1}{9}\left(1+\frac{2 b}{5}\right)+\frac{b K_{V} \delta_{i j}}{5} ; \\
& \mathcal{A}_{\alpha}=\mathcal{A}_{\alpha}^{\prime}+\mathbf{D}, \quad \mathcal{A}_{\alpha}^{\prime}=\left\{\mathcal{A}_{i j k l}^{\prime \alpha}\right\}, \quad \mathbf{D}=\left\{D_{i j k l}\right\}, \\
& \mathcal{A}_{i j k l}^{\prime \alpha}=C_{i j k l}^{\alpha} B_{1}+\frac{1}{2}\left(C_{i k j l}^{\alpha}+C_{j k i l}^{\alpha}\right) B_{2}+\frac{1}{2}\left(C_{i j p p}^{\alpha} \delta_{k l}+C_{k l p p}^{\alpha} \delta_{i j}\right) B_{3} \\
& +\frac{1}{2}\left(C_{i p j p}^{\alpha} \delta_{k l}+C_{k p l p}^{\alpha} \delta_{i j}\right) B_{4}+\frac{1}{4}\left(C_{i p k p}^{\alpha} \delta_{j l}+C_{j p k p}^{\alpha} \delta_{i l}+C_{j p l p}^{\alpha} \delta_{i k}+C_{i p l p}^{\alpha} \delta_{j k}\right) B_{5} \\
& +\frac{1}{4}\left(C_{i k p p}^{\alpha} \delta_{j l}+C_{j k p p}^{\alpha} \delta_{i l}+C_{j l p p}^{\alpha} \delta_{i k}+C_{i l p p}^{\alpha} \delta_{j k}\right) B_{6}, \\
& B_{1}=\frac{1}{9}\left(1+\frac{2 b}{5}\right)^{2}+f_{1} F_{1}+g_{1} G_{1}, \quad B_{2}=f_{1} F_{2}+g_{1} G_{2}, \quad B_{4}=f_{1} F_{4}+g_{1} G_{4}, \\
& B_{3}=\frac{2 b}{45}+\frac{4 b^{2}}{225}+f_{1} F_{3}+g_{1} G_{3}, \quad B_{5}=f_{1} F_{5}+g_{1} G_{5}, \quad B_{6}=f_{1} F_{6}+g_{1} G_{6}, \\
& D_{i j k l}=\delta_{i j} \delta_{k l} D_{1}+\frac{1}{2}\left(\delta_{i k} \delta_{j l}+\delta_{i l} \delta_{j k}\right) D_{2} \text {, } \\
& D_{1}=\left(K_{V}-\frac{2}{3} \mu_{V}\right)\left(f_{3} F_{1}+g_{3} G_{1}\right)+\mu_{V}\left(f_{3} F_{2}+g_{3} G_{2}\right)+3 K_{V}\left(f_{3} F_{3}+g_{3} G_{3}\right) \\
& +\left(K_{V}+\frac{10}{3} \mu_{V}\right)\left(f_{3} F_{4}+g_{3} G_{4}\right)+\frac{2}{3} F_{7}+\frac{4}{5} G_{7}+\frac{b^{2}}{25} K_{V}, \\
& D_{2}=2 \mu_{V}\left(f_{3} F_{1}+g_{3} G_{1}\right)+\left(K_{V}+\frac{1}{3} \mu_{V}\right)\left(f_{3} F_{2}+g_{3} G_{2}\right) \\
& +\left(K_{V}+\frac{10}{3} \mu_{V}\right)\left(f_{3} F_{5}+g_{3} G_{5}\right)+3 K_{V}\left(f_{3} F_{6}+g_{3} G_{6}\right)+\frac{2}{3} F_{8}+\frac{4}{5} G_{8}, \\
& F_{1}=-\frac{1}{15}-\frac{8 b}{105}-\frac{13 b^{2}}{315}, \quad G_{1}=\frac{43 b^{2}}{1890}, \quad F_{2}=\frac{1}{10}+\frac{4 b}{35}+\frac{16 b^{2}}{315} ， \\
& G_{2}=G_{4}=G_{6}=-\frac{4 b^{2}}{189}, \quad F_{3}=-\frac{4 b}{105}-\frac{4 b^{2}}{315}, \quad G_{3}=-\frac{b^{2}}{945} ， \\
& F_{4}=F_{6}=\frac{2 b}{35}+\frac{16 b^{2}}{315}, \quad F_{5}=\frac{1}{10}+\frac{4 b}{35}-\frac{4 b^{2}}{315}, \quad G_{5}=\frac{10 b^{2}}{189}, \\
& F_{7}=-\frac{4 b^{2}}{105} K_{V}+\frac{4 b^{2}}{63} \mu_{V}, \quad G_{7}=\frac{b^{2}}{630} K_{V}-\frac{5 b^{2}}{189} \mu_{V} ， \\
& F_{8}=\frac{b^{2}}{105} K_{V}-\frac{10 b^{2}}{63} \mu_{V}, \quad G_{8}=\frac{2 b^{2}}{63} K_{V}+\frac{5 b^{2}}{27} \mu_{V} \text {; } \\
& \overline{\mathbf{C}}_{K \alpha}=\left\{\bar{C}_{i j}^{K \alpha}\right\}, \quad \bar{C}_{i j}^{K \alpha}=S_{i j p p}^{\alpha}\left(\frac{2 b}{15}-\frac{1}{3}\right)-K_{R}^{-1} \delta_{i j}\left(\frac{4 b}{15}+\frac{1}{3}\right) ;
\end{aligned}
$$




$$
\begin{aligned}
& \overline{\mathcal{A}}_{\alpha}=\overline{\mathcal{A}}_{\alpha}^{\prime}+\overline{\mathbf{D}}, \quad \overline{\mathcal{A}}_{\alpha}^{\prime}=\left\{\overline{\mathcal{A}}_{i j k l}^{\prime \alpha}\right\}, \quad \overline{\mathbf{D}}=\left\{\bar{D}_{i j k l}\right\}, \quad \mathbf{S}^{\alpha}=\left(\mathbf{C}^{\alpha}\right)^{-1}, \\
& \overline{\mathcal{A}}_{i j k l}^{\alpha}=S_{i j k l}^{\alpha} \bar{B}_{1}+\frac{1}{2}\left(S_{i k j l}^{\alpha}+S_{j k i l}^{\alpha}\right) \bar{B}_{2}+\frac{1}{2}\left(S_{i p j p}^{\alpha} \delta_{k l}+S_{k p l p}^{\alpha} \delta_{i j}\right) \bar{B}_{4} \\
& +\frac{1}{2}\left(S_{i j p p}^{\alpha} \delta_{k l}+S_{k l p p}^{\alpha} \delta_{i j}\right) \bar{B}_{3}+\frac{1}{4}\left(S_{i p k p}^{\alpha} \delta_{j l}+S_{j p k p}^{\alpha} \delta_{i l}+S_{j p l p}^{\alpha} \delta_{i k}+S_{i p l p}^{\alpha} \delta_{j k}\right) \bar{B}_{5} \\
& +\frac{1}{4}\left(S_{i k p p}^{\alpha} \delta_{j l}+S_{j k p p}^{\alpha} \delta_{i l}+S_{j l p p}^{\alpha} \delta_{i k}+S_{i l p p}^{\alpha} \delta_{j k}\right) \bar{B}_{6}, \\
& \bar{B}_{1}=\frac{1}{9}\left(1-\frac{2 b}{5}\right)^{2}+f_{1} \bar{F}_{1}+g_{1} \bar{G}_{1}, \quad \bar{B}_{2}=f_{1} \bar{F}_{2}+g_{1} \bar{G}_{2}, \quad \bar{B}_{4}=f_{1} \bar{F}_{4}+g_{1} \bar{G}_{4}, \\
& \bar{B}_{3}=\frac{2}{9}+\frac{4 b}{45}-\frac{16 b^{2}}{225}+f_{1} \bar{F}_{3}+g_{1} \bar{G}_{3}, \quad \bar{B}_{5}=f_{1} \bar{F}_{5}+g_{1} \bar{G}_{5}, \quad \bar{B}_{6}=f_{1} \bar{F}_{6}+g_{1} \bar{G}_{6} ， \\
& \bar{D}_{i j k l}=\delta_{i j} \delta_{k l} \bar{D}_{1}+\frac{1}{2}\left(\delta_{i k} \delta_{j l}+\delta_{j k} \delta_{i l}\right) \bar{D}_{2} \text {, } \\
& \bar{D}_{1}=\left(\frac{1}{9} K_{R}^{-1}-\frac{1}{6} \mu_{R}^{-1}\right)\left(f_{3} \bar{F}_{1}+g_{3} \bar{G}_{1}\right)+\frac{1}{4} \mu_{R}^{-1}\left(f_{3} \bar{F}_{2}+g_{3} \bar{G}_{2}\right)+\frac{1}{3} K_{R}^{-1}\left(f_{3} \bar{F}_{3}+g_{3} \bar{G}_{3}\right) \\
& +\left(\frac{1}{9} K_{R}^{-1}+\frac{5}{6} \mu_{R}^{-1}\right)\left(f_{3} \bar{F}_{4}+g_{3} \bar{G}_{4}\right)+\frac{2}{3} \bar{F}_{7}+\frac{4}{5} \bar{G}_{7}+\frac{1}{9}\left(1+\frac{4 b}{5}\right)^{2} K_{R}^{-1}, \\
& \bar{D}_{2}=\left(\frac{1}{2} \mu_{R}^{-1}\left(f_{3} \bar{F}_{1}+g_{3} \bar{G}_{1}\right)+\left(\frac{1}{9} K_{R}^{-1}+\frac{1}{12} \mu_{R}^{-1}\right)\left(f_{3} \bar{F}_{2}+g_{3} \bar{G}_{2}\right)\right. \\
& +\left(\frac{1}{9} K_{R}^{-1}+\frac{5}{6} \mu_{R}^{-1}\right)\left(f_{3} \bar{F}_{5}+g_{3} \bar{G}_{5}\right)+\frac{1}{3} K_{R}^{-1}\left(f_{3} \bar{F}_{6}+g_{3} \bar{G}_{6}\right)+\frac{2}{3} \bar{F}_{8}+\frac{4}{5} \bar{G}_{8}, \\
& \bar{F}_{1}=-\frac{4}{15}-\frac{16 b}{105}-\frac{13 b^{2}}{315}, \quad \bar{G}_{1}=\frac{43 b^{2}}{1890}, \quad \bar{F}_{2}=\frac{2}{5}+\frac{8 b}{35}+\frac{16 b^{2}}{315}, \\
& \bar{G}_{2}=\bar{G}_{4}=\bar{G}_{6}=-\frac{4 b^{2}}{189}, \quad \bar{F}_{3}=\frac{22 b+28}{105}+\frac{2 b^{2}}{315}, \quad \bar{G}_{3}=-\frac{b^{2}}{945}, \\
& \bar{F}_{4}=\frac{4 b}{35}+\frac{16 b^{2}}{315}, \quad \bar{F}_{5}=\frac{2}{5}+\frac{8 b}{35}-\frac{4 b^{2}}{315}, \quad \bar{G}_{5}=\frac{10 b^{2}}{189}, \quad \bar{F}_{6}=-\frac{32 b+28}{35}-\frac{8 b^{2}}{45}, \\
& \bar{F}_{7}=\frac{b^{2}}{63} \mu_{R}^{-1}-\frac{31 b^{2}+90 b+63}{945} K_{R}^{-1}, \bar{G}_{7}=\frac{b^{2}}{5670} K_{R}^{-1}-\frac{5 b^{2}}{756} \mu_{R}^{-1}, \\
& \bar{F}_{8}=\frac{136 b^{2}+324 b+189}{945} K_{R}^{-1}-\frac{5 b^{2}}{126} \mu_{R}^{-1}, \quad \bar{G}_{8}=\frac{4 b^{2}}{1134} K_{R}^{-1}+\frac{5 b^{2}}{108} \mu_{R}^{-1} \text {; }
\end{aligned}
$$




\section{APPENDIX B}

For the lower bound $K^{L}$, we have the following particular expressions of its constituent two-index-notation matrices

$$
\begin{aligned}
& \overline{\mathbf{C}}_{K}=\left\{\bar{C}_{i j}^{K}\right\} \quad(\text { symmetric } 3 \times 3 \text { matrix }), \\
& \bar{C}_{11}^{K}=\left(S_{11}+S_{12}+S_{13}\right)\left(\frac{2 b}{15}-\frac{1}{3}\right)-\frac{1}{K_{R}}\left(\frac{4 b}{15}+\frac{1}{3}\right) \\
& \bar{C}_{22}^{K}=\left(S_{22}+S_{12}+S_{23}\right)\left(\frac{2 b}{15}-\frac{1}{3}\right)-\frac{1}{K_{R}}\left(\frac{4 b}{15}+\frac{1}{3}\right) \\
& \bar{C}_{33}^{K}=\left(S_{33}+2 S_{13}\right)\left(\frac{2 b}{15}-\frac{1}{3}\right)-\frac{1}{K_{R}}\left(\frac{4 b}{15}+\frac{1}{3}\right), \bar{C}_{12}^{K}=\bar{C}_{13}^{K}=\bar{C}_{23}^{K}=0 ; \\
& \overline{\mathcal{A}}=\left\{\bar{S}_{p q}^{A}\right\}, \quad \bar{S}_{p q}^{A}=\bar{S}_{p q}^{\prime A}+\bar{D}_{p q} \quad(\text { symmetric } 6 \times 6 \text { matrices }), \\
& \bar{S}_{11}^{\prime A}=S_{11}\left(\bar{B}_{1}+\bar{B}_{2}\right)+\left(S_{11}+S_{12}+S_{13}\right)\left(\bar{B}_{3}+\bar{B}_{6}\right) \\
&+\left(S_{11}+\frac{1}{4} S_{55}+\frac{1}{4} S_{66}\right)\left(\bar{B}_{4}+\bar{B}_{5}\right)=\bar{S}_{22}^{\prime A}, \\
& \bar{S}_{33}^{\prime A}=S_{33}\left(\bar{B}_{1}+\bar{B}_{2}\right)+\left(S_{33}+2 S_{13}\right)\left(\bar{B}_{3}+\bar{B}_{6}\right)+\left(S_{33}+\frac{1}{2} S_{44}\right)\left(\bar{B}_{4}+\bar{B}_{5}\right), \\
& \bar{S}_{12}^{\prime A}= S_{12} \bar{B}_{1}+\frac{1}{4} S_{66} \bar{B}_{2}+\left(S_{11}+S_{12}+S_{13}\right) \bar{B}_{3}+\left(S_{11}+\frac{1}{4} S_{44}+\frac{1}{4} S_{66}\right) \bar{B}_{4}, \\
& \bar{S}_{13}^{\prime A}= S_{13} \bar{B}_{1}+\frac{1}{4} S_{55} \bar{B}_{2}+\left(S_{11}+S_{12}+3 S_{13}+S_{33}\right) \frac{1}{2} \bar{B}_{3} \\
&+\left(S_{11}+S_{33}+\frac{3}{4} S_{44}+\frac{1}{4} S_{66}\right) \frac{1}{2} \bar{B}_{4}=\bar{S}_{23}^{\prime A}, \\
& \bar{D}_{11}^{\prime A}= \bar{D}_{22}=\bar{D}_{66} \bar{B}_{1}+\left(S_{12}+\frac{1}{4} S_{66}\right) 2 \bar{B}_{2}+\left(2 S_{11}+\frac{1}{2} S_{44}+\frac{1}{2} S_{66}\right) \bar{B}_{5} \\
&+2\left(S_{11}+S_{12}+S_{13}\right) \bar{B}_{6}, \\
& \bar{S}_{44}^{\prime A}= S_{44} \bar{B}_{1}+\left(S_{23}+\frac{1}{4} S_{44}\right) 2 \bar{B}_{2}+\left(S_{11}+S_{33}+\frac{3}{4} S_{44}+\frac{1}{4} S_{66}\right) \bar{B}_{5} \\
&+\left(S_{11}+S_{12}+3 S_{13}+S_{33}\right) \bar{B}_{6}=\bar{S}_{55}^{\prime A}, \\
& \bar{S}_{63} \bar{D}_{1} ; \bar{D}_{44}=\bar{D}_{55}=\bar{D}_{66}=2 \bar{D}_{2},
\end{aligned}
$$

where $\bar{B}_{1}, \ldots, \bar{B}_{6}$ and $\bar{D}_{1}, \bar{D}_{2}$ are defined Appendix A.

$$
\overline{\mathcal{A}}^{-1}=\left\{\bar{C}_{p q}^{A}\right\}=\left\{\bar{S}_{p q}^{A}\right\}^{-1} \quad(6 \times 6 \text { symmetric matrix }) .
$$

Then

$$
\begin{aligned}
& \overline{\mathcal{A}}^{-1}: \overline{\mathbf{C}}_{K}=\overline{\mathbf{C}}_{K}: \overline{\mathcal{A}}^{-1}=\left\{\bar{C}_{K i j}^{A C}\right\} \quad(3 \times 3 \text { symmetric matrix }), \\
& \bar{C}_{K 11}^{A C}=\left(\bar{C}_{11}^{A}+\bar{C}_{12}^{A}\right) \bar{C}_{11}^{K}+\bar{C}_{13}^{A} \bar{C}_{33}^{K}=\bar{C}_{K 22}^{A C}, \\
& \bar{C}_{K 33}^{A C}=\bar{C}_{33}^{A} \bar{C}_{33}^{K}+2 \bar{C}_{13}^{A} \bar{C}_{11}^{K}, \quad \bar{C}_{K 12}^{A C}=\bar{C}_{K 13}^{A C}=\bar{C}_{K 23}^{A C}=0,
\end{aligned}
$$


and

$$
\overline{\mathbf{C}}_{K}: \overline{\mathcal{A}}^{-1}: \overline{\mathbf{C}}_{K}=\bar{C}_{K}^{C A C}=2 \bar{C}_{11}^{K} \bar{C}_{K 11}^{A C}+\bar{C}_{33}^{K} \bar{C}_{K 33}^{A C} .
$$

Now one has

$$
\begin{gathered}
\left\langle\overline{\mathcal{A}}_{\alpha}^{-1}\right\rangle_{\alpha}=\mathbf{T}\left(\mathcal{K}_{V}\left(\left\{\bar{C}_{p q}^{A}\right\}\right), \mathcal{M}_{V}\left(\left\{\bar{C}_{p q}^{A}\right\}\right)\right) \\
\left\langle\overline{\mathcal{A}}_{\alpha}^{-1}\right\rangle_{\alpha}^{-1}=\mathbf{T}\left(\frac{1}{9} \mathcal{K}_{V}^{-1}\left(\left\{\bar{C}_{p q}^{A}\right\}\right), \frac{1}{4} \mathcal{M}_{V}^{-1}\left(\left\{\bar{C}_{p q}^{A}\right\}\right)\right), \\
\left\langle\overline{\mathcal{A}}_{\alpha}^{-1}: \overline{\mathbf{C}}_{K \alpha}\right\rangle_{\alpha}=\left\langle\overline{\mathbf{C}}_{K \alpha}: \overline{\mathcal{A}}_{\alpha}^{-1}\right\rangle_{\alpha}=\mathbf{I} \frac{1}{3}\left(2 \bar{C}_{K 11}^{A C}+\bar{C}_{K 33}^{A C}\right) .
\end{gathered}
$$

